\title{
THE WAY OF SEARCHING LOST AIRCRAFT
}

\author{
Jian Chen \\ North China Electric Power University Baoding
}

Keywords: Crash location, motion of airframe, focused searching area

\begin{abstract}
Recall the lost Malaysian flight MH370. To build a generic mathematical model that could assist "searchers" in planning a useful search for a lost plane feared to have crashed in open water. Our model has two parts, of which the first part is to find the location where the aircraft is more likely to fall down, the second part is to simulate the real time motion of the aircraft wreck.
\end{abstract}

\section{Introduction}

Since the airplane is invented by Wright Brothers, it has greatly improved people's traffic efficiency and produced large quantity of fortunes. Now the flight has become an indispensable transport mode. However, the frequent crash accidents occurred recently have greatly scared many people. The key problem to maximally save people's life after the crash is how to successfully search and find the lost plane as quickly as possible. It is also significant to rebuild people's confidence and enthusiasm on taking a flight travel.

By far, though the international community has established the safety system and studied the offshore drifting models, the more proper method for the searching work, especially the way to distinguish the probability distribution of the location, is still need to be developed.

\section{The probable Crashed Area Calculation}

\subsection{Movement equation of the aircraft}

We assume that the beginning time, when the plane is treated as lost, is the end of the last communication during the flight from point $\mathrm{A}$ to point $\mathrm{B}$. To investigate the movement of the aircraft after it lost, we take the aerodynamic drag factor into account and use the following motion equation.[1]

$$
\left\{\begin{array}{l}
x=\frac{m v_{0}}{k}\left(1-e^{-\frac{k}{m} t}\right) \\
y=\frac{m g}{k} t+\frac{m^{2} g}{k^{2}}\left(e^{-\frac{k}{m} t}-1\right)
\end{array}\right.
$$

where $x$ is the aircraft's flight distance in the horizontal direction,

$y$ is the falling height of the plane,

$m$ is the mass of the aircraft,

$g$ is the gravity acceleration,

$k$ is the aerodynamic drag factor,

$v_{0}$ and $h_{0}$ are respectively the original flying speed and the height of the plane when it begins to be lost, of which the initial height $h_{0}$ will be variable due to different types of planes, and $t_{0}$ is the time that the aircraft remains keeping flight after it lost but before it begins to fall.

It is sensible that we calculate the maximum flight radius of the aircraft after it lost to estimate the major downed positions. Generally, the maximum radius depends on the types of the aircraft. The higher the plane is, the larger the radius will be.

\subsection{Analysis on Poisson Distribution}

To analyze the crash process more precisely, we take the general condition as the research object. 
Since the lost aircraft crashes into the ocean in an uncertain direction, we treat the distribution probability of the downed location as a Poisson distribution around the lost point where the last communication ends. Meanwhile, we use the discrete points to model the distribution probability. The district where the points are denser is the very location where the plane is more likely to fall down. As shown in Figure 1.

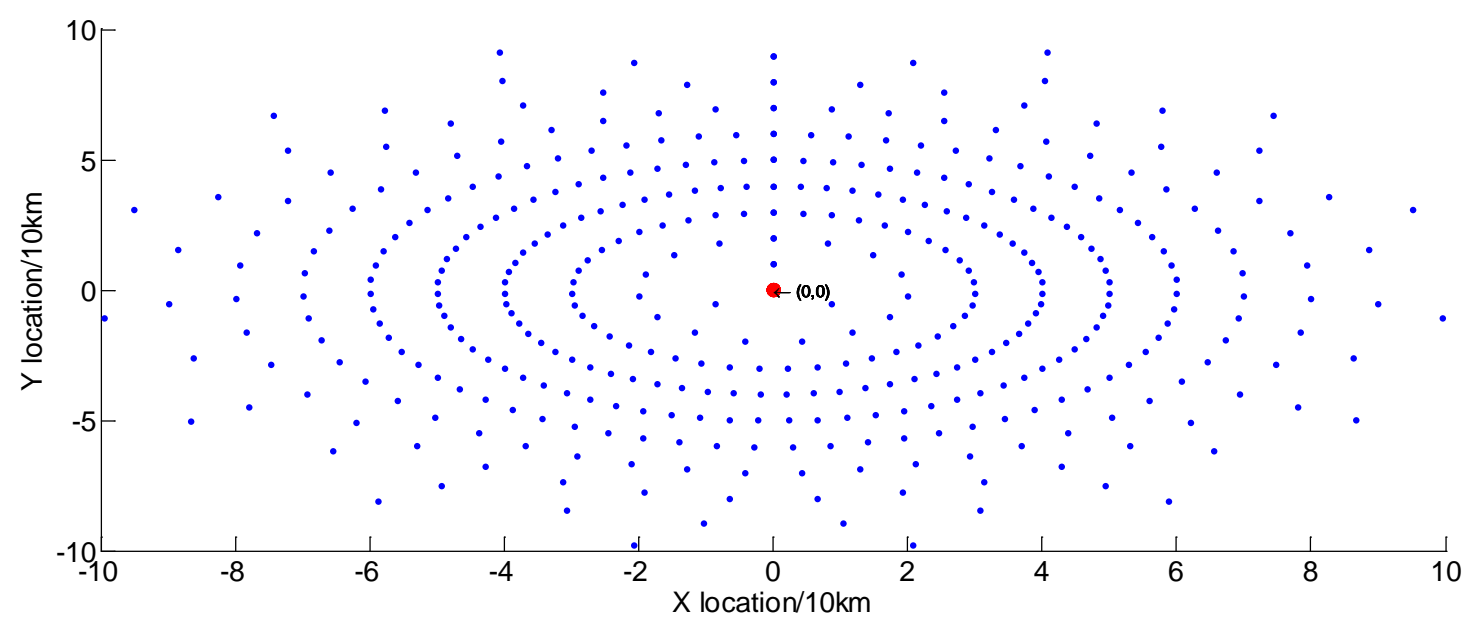

Figure 1.The distribution probability of the downed location

In the case that the drop aircraft still has power, it will keep a uniform motion in the horizontally forward direction.. And the calculation can be still carried out with the above model, only adjusting the reverse acceleration to zero. Therefore, we only focus on the former condition.

\section{Motion Analysis of the Aircraft wreck}

The motion of the lost plane will be affected mainly by the wind stress, the ocean current, and the Coriolis force, of which the Coriolis force will be always on work. The motion of the plane after downed to the water can be divided into three steps.

Step 1: The plane will be float on the water for a short time during the submersion process. In this case, the wind stress and the supernatant water current are the major causes to affect the motion of the aircraft.

Step 2: When the plane is entirely submerged in the water but does not touch the seabed, the ocean current which is one kind of steady flow influenced by the factors such as wind stress, tidal force, density contrast, etc., will be the most primary cause to make the downed plane drifted.

Step 3: when the aircraft has touched the seabed, it is thought that the airframe will be kept stable due to the very tiny velocity of the ocean current.

\subsection{Real time model of ocean current}

The existing ocean current model [2] has some unavoidable errors due to the vast area of the ocean and the unidentified factors. These errors are acceptable for a rough resolution in the space and time, while for the lost plane search they will cause serious influences. To obtain a more precise result about the effect of the ocean current on the downed plane, we set up a real time ocean current model [3] linking to an actual database. Further, we also take the wind force and the Coriolis Effect into account to calculate the motion of the aircraft in the ocean.

The tide [4], which is a periodic seawater movement induced by the gravitation from the sun and the moon , is the main cause to make the ocean current change uncertainly. In a certain location, the tide usually includes several components. And each tide component has its own frequency. Therefore, the ocean current velocity can be expressed by a series of sine waves and cosine waves which contain the tide components' frequencies and the space information at the same time [5], as 


$$
u_{m}(x, t)=u_{0}(x)+\sum_{i=1}^{N}\left[g_{i}(x) \cos \left(\omega_{i} t\right)\right]+\sum_{i=1}^{N}\left[h_{i}(x) \sin \left(\omega_{i} t\right)\right]
$$

where $N$ is the number of the tide components in a certain location,

$\boldsymbol{x}$ is the position where the aircraft is thought to be lost,

$\boldsymbol{u}_{0}(\boldsymbol{x})$ is the average velocity of the ocean current,

$\boldsymbol{g}_{\boldsymbol{i}}(\boldsymbol{x})$ and $\boldsymbol{h}_{\boldsymbol{i}}(\boldsymbol{x})$ are the position functions of the downed plane in the horizontal direction and the vertical direction, respectively,

$\mathrm{Di}(\boldsymbol{x})$ is the coefficient of different depth under the current speed.

The exact values of $N$ and $x$ can be obtained on line from the linking database. And then $\boldsymbol{u}_{\boldsymbol{0}}(\boldsymbol{x}), \boldsymbol{g}_{\boldsymbol{i}}(\boldsymbol{x})$ and $\boldsymbol{h}_{\boldsymbol{i}}(\boldsymbol{x})$ can be calculated by

$$
\left\{\begin{array}{l}
u_{0}(x)=\sum_{j=1}^{M} \theta_{1, j} \phi_{j}(x) \\
g_{i}(x)=\sum_{j=1}^{M} \theta_{2 i, j} \phi_{j}(x) \\
h_{i}(x)=\sum_{j=1}^{M} \theta_{2 i+1, j} \phi_{j}(x) \\
D_{i}(x)=\sum_{j=1}^{M} \theta_{2 i-1, j+1} \phi_{j}(x) \\
\phi_{j}(x)=e^{-\frac{\left(x-c_{j}\right)^{2}}{2 \sigma^{2}}}
\end{array}\right.
$$

where $\Phi_{j}(x)$ is a radial basis function,

$\sigma$ is the width of the radial basis function,

$C_{j}$ is the center of $\Phi_{j}(x)$,

$\theta_{i j}$ is a coefficient of $\Phi_{j}(x)$,

and $M$ is the function number of $\Phi_{j}(x)$.

Then we obtain the final formula of the ocean current as

$$
\left\{\begin{array}{l}
u_{m}(x, t)=\sum_{j=1}^{M} \alpha_{j}(t) \phi_{j}(x) \\
\alpha_{j}(t)=\theta_{1, j}+\sum_{i=1}^{N} \theta_{2 i, j} \cos \left(\omega_{i} t\right)+\sum_{i=1}^{N} \theta_{2 i+1, j} \sin \left(\omega_{i} t\right)
\end{array}\right.
$$

According to Eq.(4), we are able to get a accurate value of the ocean velocity by only inputting the geographic location, as indicated in Figure 2. In this section, it is important to note that the detailed location is shifted because the airframe will be pushed away by the ocean current. Moreover, the Coriolis force and the wind force actually affect the motion of the plane as well. But the wind force is only on work in Step 1 (see the following motion analysis section, we divide the motion of the plane into three steps, of which Step 1 is the submersion process, Step 2 is the process entirely under the seawater until touching the seabed, and Step 3 is that the airframe has touched the seabed.), while the Coriolis force acts all the time. Then it is needed for us to take iterative computations and repeated database indexing performances, so that the real-time position and the motion orbit of the plane can be obtained. 


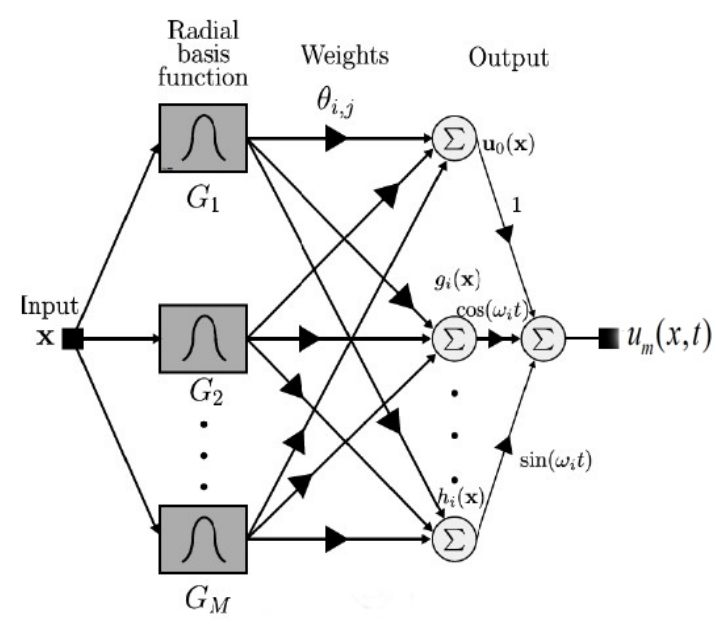

Figure 2. Structure of the tidal model

\section{Summary}

We set up the movement equations to analyze the aircraft's movement in the air, and use the Poisson distribution to establish the positions where the lost plane is most likely to fall down. Finally we obtained a probability distribution figure composed by many discrete points. The locations where the points are denser are the very places that the lost plane is most likely to crash in.

There are actually three steps during the motion process of the plane wreck: 1) floating on the seawater, 2) drifting under the seawater, and 3) arriving at the seabed. The motion will be affect by the ocean current, the wind force, and the Coriolis Effect at the same time. We study on the motion in the horizontal direction and the vertical direction, respectively, and find that, 1) in the horizontal direction the airframe will be mainly drifted along the ocean current, 2) in the vertical direction the airframe will be firstly accelerated and then under a uniform motion until it touches the seabed, 3) when the plane wreck touches the seabed, it will be generally kept stable.

\section{References}

[1]. R. Pawlowicz, B. Beardsley, and S. Lentz, "Classical tidal harmonic analysis including error estimates in MATLAB using T_TIDE,” Computers \& Geosciences, vol. 28, no. 8, pp. 929-937, 2002.

[2]. Shao-rong Feng, Wen-jun Xiao, An Improved DBSCAN Clustering Algorithm, Journal of China University of Mining\& Technology, Vol.37 No.1, Jan. 2008.

[3]. Sheng-wei Xing, Research on Global Optimization Model and Simulation of Joint Aeronautical and Maritime Search, Dalian Maritime University for the degree of Doctor of Engineering, September 2012.

[4]. Long DI, Qi-di Zhang, The Study on the Track of Horizontal Parabola in Air Resistance, No.1, 2014 (in Chinese).

[5]. Long DI, Qi-di Zhang, The Study on the Track of Horizontal Parabola in Air Resistance, No.1, 2014 (in Chinese). 